\title{
Factores que predicen la permanencia estudiantil: Análisis en un escenario de educación técnica
}

\author{
Predictive factors in student retention: \\ Analysis in a technical education scenario
}

DOI: http://dx.doi.org/10.17981/cultedusoc.11.1.2020.02

Recibido: 09/09/2019. Aceptado: 14/01/2020. Publicado: 20/02/2020

Juan Carlos Olarte Moyano $\odot$

Politécnico Internacional. Bogotá, D.C. (Colombia)

juacolarte@gmail.com

Para citar este artículo:

Olarte, J. (2020). Factores que predicen la permanencia estudiantil: Análisis en un escenario de educación técnica. Cultura, Educación y Sociedad, 11(1). 25-38. DOI: http://dx.doi.org/10.17981/cultedusoc.11.1.2020.02

\section{Resumen}

El objetivo de este estudio es examinar las variables de ingreso de los estudiantes que pueden predecir la permanencia estudiantil mediante el relacionamiento de siete variables ampliamente estudiadas en la literatura y de interés para el contexto de estudio. El modelo propuesto relaciona la edad, el género, el estado civil, la ciudad de residencia, el estrato, los resultados de las pruebas de estado y el tipo de colegio donde terminó los estudios de secundaria como variables explicativas de la permanencia estudiantil. Para el análisis se utilizó el modelo de Cox de riesgos proporcionales ya que incluye las ventajas de un modelo de duración al tener en cuenta el tiempo de ocurrencia de un evento y permite incluir variables de correlación. La investigación arrojó relaciones estadísticamente significativas en las variables de edad y estrato social como predictores de la permanencia estudiantil.

Palabras clave: Deserción escolar; fracaso escolar; educación superior; educación técnica

\begin{abstract}
The objective of this study is to examine the students' entry variables, that could predict the student retention by the relation of seven variables thoroughly studied in the literature, which are also considered of interest for the assigned context. The proposed model relates age, gender, marital status, city of residence, social stratum, results in academic tests, and the kind of school where students finished high school as the explanatory variables of the student's retention. For the analysis, it was implemented the Cox proportional hazards model, due to the fact that it includes the advantages of a survival model, taking into account the time until the occurrence of a well-defined event, and it allows to include predictors or explanatory variables. The research brought statistically significant relations in the variables of age and social stratum like predictors of the student's retention. Keywords: Student dropout; student attrition; higher education; technical education
\end{abstract}




\section{INTRODUCCIÓN}

La deserción universitaria es una problemática que se presenta a nivel mundial y cuyos indicadores se convierten en un escenario de análisis de alta importancia (Patiño y Cardona, 2012) (Rue, 2014). El crecimiento de la deserción universitaria se ha manifestado en las últimas décadas llegando a unas tasas considerables de abandono. Las mediciones confirman que alrededor de la mitad de las personas que ingresan a un programa universitario no culminan sus estudios.

Por ejemplo, las tasas de abandono en España, Francia, Austria y Estados Unidos oscilan entre $30 \%$ y $50 \%$ y se obtienen cifras similares en Latinoamérica y otro gran número de países. Sin embargo, son algo más bajas en Alemania (20-25\%), Suiza (7\%$30 \%$ ), Finlandia (10\%) y Países Bajos (20\%-30\%) que sin duda refleja los esfuerzos de estos países por lograr que sus estudiantes obtengan su título. Otro dato importante es que la mayor pérdida de estudiantes se da en los primeros periodos académicos con un promedio entre $25 \%$ y $35 \%$ de los estudiantes que ingresan a un programa universitario. Estos indicadores demuestran una necesidad puntual en el escenario de la educación, que se ha venido estudiando en las universidades (Huesca, Ramírez y Castaño, 2007).

Para el caso de Colombia, la realidad en cuanto a la permanencia de los estudiantes en educación superior no difiere mucho del escenario internacional. Desde el momento en que el Ministerio de Educación Nacional viene realizando una medición por cohortes a nivel nacional de manera regular y homogénea, las tasas de deserción del sistema se han ubicado entre el $45 \%$ y el $50 \%$, lo que significaría que cerca de uno de cada dos estudiantes que ingresan al sistema no culmina sus estudios. Si bien esta cifra puede ser alarmante no es ajena a la dinámica latinoamericana en materia de educación superior, donde sólo países como Argentina y Cuba presentaban para 2007 índices inferiores (Ministerio de Educación Nacional, 2009).

Las mediciones más recientes del Ministerio de Educación Nacional de Colombia (2009) hablan de una deserción para todo el nivel de educación superior del 49\%, tasa que incluye la deserción en el nivel universitario, técnico y tecnológico. De manera general se destaca una menor deserción en instituciones oficiales (45.3\%) frente a 52.1\% en las instituciones privadas. Así mismo se reconoce una menor deserción en el nivel universitario con una tasa acumulada del 44.2\%, siendo aún menor en instituciones oficiales (39.9\%) frente a $47.7 \%$ en privadas. Las mayores tasas están en el nivel de formación técnica profesional con una deserción del $60.6 \%$, seguidas por el nivel tecnológico con $52.6 \%$.

Este estudio se desarrolla en el Politécnico Internacional que es Institución Colombiana de nivel Técnico Profesional. Los indicadores de deserción de la Institución también reflejan la realidad del país y confirman la problemática que las universidades viven con el abandono por parte de sus estudiantes. Específicamente, el Politécnico Internacional tiene un promedio de deserción del 60\% por lo que, ni la mitad de los estudiantes logran obtener su título. Adicionalmente, se presenta que en promedio el $35 \%$ de los estudiantes abandonan su carrera en el primer periodo académico y el 50\% ya ha desertado para el primer año de estudioº 


\section{Marco DE REFERENCIA}

Múltiples estudios han permitido el avance en la comprensión de las variables que influyen en la decisión de abandono por parte de un estudiante. En general, los estudios sugieren que las variables que pueden explicar este fenómeno incluyen los modelos educativos, el costo de los programas académico y las condiciones personales y del entorno social del estudiante (Patiño \& Cardona, 2012).Las investigaciones muestran que los estudiantes que desertan, comparados con aquellos que permanecen en la universidad, tienden a tener menores notas académicas en promedio y padres con menor educación e inferiores ingresos. También se ha estimado que los estudiantes con un mayor nivel de interacción con el claustro de profesores y con otros estudiantes tienen menor probabilidad de abandonar, pero es muy posible que tal interacción esté relacionada con múltiples factores que influyen en la probabilidad de desertar (Giovagnoli, 2002).

Sin duda, los factores económicos se mencionan con gran frecuencia como uno de los factores que explica la permanencia estudiantil (Ishitani \& DesJardins, 2002; Huesca \& Castaño, 2007). Por lo anterior, se espera que personas con dificultades en obtener unos ingresos suficientes, tengan una probabilidad menor de terminar sus estudios. De esta forma, varias de las estrategias para prevenir la deserción se enfocan en la creación de sistemas de financiación bien sea por medio de becas o descuentos en el valor de la matrícula a un programa académico. Sin embargo, Tinto (1993) afirma que hay pocas pruebas directas que apoyen la afirmación de que la situación económica, por sí misma, sea un determinante significativo en el proceso de deserción. Señala Tinto que, aunque los estudiantes desertores atribuyan a menudo a las dificultades financieras las razones de su abandono, estas afirmaciones ocultan los motivos básicos de la deserción.

Por su lado, Tinto (1993) afirma que la deserción surge por la dificultad que tienen algunos estudiantes para lograr una adecuada inmersión en la vida universitaria. Son el tipo de interacciones que logra constituir el estudiante lo que determina la permanencia en la universidad. De esta forma el apoyo y aceptación que viva el estudiante por parte de su entorno determina el nivel de arraigo a los estudios que permite superar los obstáculos que se pueden presentar durante el desarrollo del proceso educativo. Lo anterior también está alineado con la teoría de que la transición entre la educación media y la superior debe ser objeto de análisis ya que en este proceso se determina la adaptación a la vida universitaria (Huesca \& Castaño, 2007). A partir de esto se han creado varias estrategias que giran en torno a variables institucionales como la misión, la visión, el modelo educativo, el ambiente educativo y el perfil profesional y ocupacional del programa, así como los procesos pedagógicos (Ministerio de Educación Nacional, 2015).

Ahora bien, esta investigación acoge los estudios que han incorporado factores explicativos relacionados con variables propias del sujeto en el momento de la incursión en la vida universitaria (Díaz, 2009; González, Álvarez, Cabrera \& Bethencourt, 2007). En esta línea, los factores que se consideran para explicar la deserción estudiantil universitaria, incluyen variables como la edad, estado civil, el tipo de colegio secundario, ingreso económico, nivel educativo de los padres, condiciones laborales de entrada, lugar de origen, con quien vive, número de hijos, entre otros. Por ejemplo, algunos estudios encontraron una relación positiva entre el nivel educacional de los padres y los comportamientos de las ge- 
neraciones anteriores del estudiante con las tasas de permanencia Pascarella y Terenzini (1983), (Gold, 1995) y Abarca y Sánchez (2005).

Otras investigaciones también conducen a entender como las variables personales de ingreso de los estudiantes pueden relacionarse con la probabilidad de la permanencia en la universidad. Por ejemplo, la probabilidad de deserción es mayor para estudiantes que trabajan ya que el rendimiento es menor y disminuye con el número de horas trabajadas (Diaz, 2009). Según Giovagnoli (2002), ha detectado una relación positiva pero marginalmente decreciente entre la edad de ingreso y el riesgo de deserción. En cuanto al género de los estudiantes predomina un mayor riesgo de deserción para los hombres comparado con las mujeres (Herrero, Merlino, Ayllon y Escanes, 2013) .También los resultados en los exámenes estandarizados o de ingreso permiten predecir el éxito de un estudiante en los estudios superiores (Huesca \& Castaño, 2007). Finalmente, otros aspectos como un bajo estrato social, vivir solo, tener una experiencia universitaria previa y obtener un bajo rendimiento en pruebas estandarizadas demuestran en otros estudios su relación con el aumento del riesgo en el abandono de los estudios universitarios (Herrero, Merlino, Ayllon y Escanes, 2013).

Además de las variables anteriormente mencionadas Enciso, Córdoba y Romero (2016) informan que las universidades colombianas tienen pocas adaptaciones curriculares que ayuden a la permanencia y graduación de estudiantes con discapacidades visuales, auditivas, físicas y cognitivas, lo cual afecta su proceso de aprendizaje dentro de las instituciones y su posible deserción.

Sumando a lo anterior, los esfuerzos por definir las causales de la decisión de abandono de su carrera por parte de un estudiante tienen como fin último la formulación de estrategias que puedan prevenir esta situación y mejorar las tasas de retención en las instituciones de educación superior. De acuerdo a Patiño y Cardona (2012), para que las instituciones puedan trabajar de forma preventiva en la deserción de los estudiantes deben seguir avanzando en la comprensión de los tipos de abandono y la validación permanente de prácticas que atiendan la problemática. Una de las conclusiones del autor es que generar unas condiciones institucionales, académicas y pedagógicas adecuadas para prevenir la deserción de los estudiantes, "implican adecuar la metodología, ampliar la información, realizar seguimientos y sistematizar dicha experiencia para intervenir eficientemente en esta problemática y así disminuir este fenómeno" (Patiño \& Cardona, 2012, p. 18).

Por su parte, Rue (2014) propone diez líneas de acción para reducir el abandono de los estudiantes que se mencionan a continuación:

1. Realizar un diagnóstico profundo y detallado para comprender las variables que definen las causas de abandono.

2. Establecer políticas y directrices institucionales que permitan establecer las actuaciones desde un marco normativo en pro de la permanencia de los estudiantes.

3. Promover estudios que potencien el aprendizaje de los estudiantes con base en datos existentes relacionados con los estudiantes y que permitan tomar decisiones objetivas. 
4. Revisar los diseños curriculares para aumentar la relevancia y pertinencia de los programas permanentemente.

5. Realizar seguimiento del currículo operativo para garantizar la adecuada implementación y formulación de acciones oportunas.

6. Mejorar las experiencias de aprendizaje para aumentar la comprensión profunda y significativa del conocimiento.

7. Incluir nuevas opciones metodológicas facilitadoras del aprendizaje que promuevan la autorregulación y la inclusión de las tecnologías de la información.

8. Promover acciones de tutorización para mejorar el acompañamiento de los estudiantes.

9. Creación de unidades dedicadas al servicio del estudiante.

10. Incentivar la motivación desde los profesores.

En definitiva, la prevención de la deserción estudiantil es una problemática con una alta relevancia de atención por sus efectos en cualquier población. Se define que las variables que intervienen en este escenario son múltiples y con diferentes connotaciones de acuerdo al contexto de actuación. Asimismo, el primer paso para la implementación de estrategias efectivas es la caracterización de las condiciones iniciales y el diagnóstico de las causas reales que determinan la decisión de abandono. Por último, las estrategias derivadas deben ser globales, alineadas con directrices institucionales y que se operen desde las aulas de clase donde las realidades de los estudiantes se vuelven explícitas y las acciones llegan a ser más efectivas. Al respecto, Luque y Marín (2001), destacan la necesidad de que el sistema educativo genere ambientes de aprendizaje innovadores, donde se articule la teoría y la práctica, de manera tal que el aprendizaje encuentre sentido. De generarse esta situación se infiere una mayor motivación por parte del estudiante para permanecer en la escuela.

\section{MetodolóGia}

\section{Objetivo e hipótesis}

El objetivo de este estudio es validar la correlación existente entre las variables contempladas para el análisis y su efecto sobre la deserción estudiantil como variable independiente. Se espera que los resultados permitan identificar aspectos clave de los estudiantes en el momento del ingreso a la vida universitaria y que puedan prever su intención de abandono en algún momento de su carrera. El poder identificar estas características podría permitir a la institución la formulación de estrategias focalizadas y relevantes para abordar la problemática de la permanencia estudiantil.

Para cumplir el objetivo, este trabajo aplica un modelo de duración para estimar la permanencia esperada de los estudiantes que ingresan a la Institución. En el modelo se incluyen algunas variables de ingreso de los estudiantes para poder determinar aquellas que tienen un efecto significativo sobre la permanencia de los estudiantes. 


\section{Variables incluidas en el modelo}

En primer lugar, se consideran los datos de permanencia estudiantil donde se definen dos variables indispensables para el modelo: el tiempo de permanencia de los estudiantes que para nuestro caso está medido en el número de ciclos académicos que el estudiante cursó y una variable categórica que indica si el estudiante desertó (1: desertó, 0: no desertó).

En cuanto a las variables explicativas del fenómeno se consideran características individuales de cada estudiante en el momento de ingreso a la Institución. Los aspectos identificados responden a una selección por conveniencia a partir de las percepciones y análisis de la información que la Institución ha desarrollado y de las cuáles se han podido hacer algunas inferencias preliminares. Específicamente las variables consideradas son: edad, género, estado civil, ciudad de residencia, estrato socioeconómico, resultados de la prueba Saber $11^{2}$ y el tipo de colegio del cual proviene el estudiante. Para el análisis todas las variables son consideradas categóricas donde se asigna 1 al aspecto que se quiere incluir en el modelo y 0 que representa la ausencia de la característica seleccionada. En la Tabla 1 se presentan las definiciones incluidas para el modelo.

TABLA 1.

Definición de las variables incluidas en el modelo.

\begin{tabular}{ll}
\multicolumn{1}{c}{ Variable } & \multicolumn{1}{c}{ Definición } \\
Edad & 1: Cuartil 1 0: Cuartil 2-3-4 \\
Género & 1: Femenino 0: Masculino \\
Estado Civil & 1: Soltero 0: Otro estado civil \\
Ciudad de Residencia & 1: Bogotá 0: Otra Ciudad \\
Estrato & 1: Estrato 1 y2 0: Otro estrato \\
Resultados Saber11 & 1: Cuartil 1 0: Cuartil 2-3-4 \\
Tipo de colegio & 1: Público 0: Privado \\
\hline
\end{tabular}

Fuente: Elaboración propia.

Para el caso de la edad y los resultados de la prueba Saber 11 se analizan, incluyendo como aspecto de incidencia, los datos que corresponden al primer cuartil de toda la información de los estudiantes. Para el caso de la edad el cuartil 1 recoge los estudiantes con edad entre 15 a 18 años, y para el caso de Saber 11 el cuartil 1 incluye los estudiantes con un puntaje inferior a 280.

Dado la anterior, las hipótesis del estudio están dirigidas a valorar la relación de las variables de ingreso incluidas con el tiempo que un estudiante permanece en su carrera. En la Tabla 2 se describen las hipótesis planteadas. 
TABLA 2.

Hipótesis propuestas en relación con las variables de ingreso y la permanencia estudiantil

\begin{tabular}{ll}
\hline Hipótesis & \multicolumn{1}{c}{ Descripción } \\
Ho1 & La permanencia no depende de la edad de los estudiantes. \\
Ho2 & La permanencia no depende del género de los estudiantes. \\
Ho3 & La permanencia no depende del estado civil de los estudiantes. \\
Ho4 & La permanencia no depende de la ciudad de residencia de los estudiantes. \\
Ho5 & La permanencia no depende del estrato de los estudiantes. \\
Ho6 & La permanencia no depende de los resultados de la prueba Saber 11 de los estudiantes. \\
Ho7 & La permanencia no depende del tipo de colegio donde estudiaron los estudiantes. \\
\hline
\end{tabular}

Fuente: Elaboración propia.

\section{Descripción de la muestra}

Para el análisis se consideran los programas académicos de igual duración entre los que se encuentran: Comercio y Negocios Internacionales, Contabilidad y Finanzas, Desarrollo de Software, Gastronomía y Hotelería. Se trabaja con la totalidad de los estudiantes que iniciaron en el primer ciclo académico de 2014 de los programas enunciados. Los datos que fundamentan el análisis corresponden a aquellos estudiantes cuya información se tiene de todas las variables consideradas para el estudio. En la Tabla 3 se muestra el total de la población, los estudiantes que se incluyen en el análisis y el porcentaje de muestra obtenido.

TABLA 3.

Muestra de estudiantes para el análisis de permanencia

\begin{tabular}{lllc}
\hline \multicolumn{1}{c}{ Programa Técnico Profesional } & No. Estudiantes Nuevos & No. Estudiantes que cumplen & $\begin{array}{c}\% \\
\text { muestra }\end{array}$ \\
Comercio y Negocios Internacionales & 288 & 151 & $52 \%$ \\
Contabilidad y Finanzas & 116 & 69 & $59 \%$ \\
Desarrollo de Software & 39 & 19 & $49 \%$ \\
Gastronomía & 676 & 287 & $42 \%$ \\
Hotelería & 208 & 107 & $51 \%$ \\
Total & 1327 & 633 & $47 \%$ \\
\hline
\end{tabular}

Fuente: Elaboración propia.

Como información inicial se presentan en la Tabla 4 las características generales de la población analizada en cuanto a las variables de interés. En general, los estudiantes incluidos en el estudio, que representan la población de la institución en el momento de su ingreso, son en su mayoría solteros (90,84\%), pertenecen a los estratos 1-2-3 (93,99\%) y residen en la ciudad de atención de la Institución $(89,10 \%)$. 
TABLA 4.

Características generales de la población estudiada.

\begin{tabular}{|c|c|}
\hline Variable & Descripción \\
\hline Edad & $\begin{array}{l}\text { Media: } 21 \text { Mínimo: } 15 \text { Máximo: } 52 \\
\text { Estudiantes entre 15-18 años: } 37 \%\end{array}$ \\
\hline Género & Mujeres: 47\% Hombres: 53\% \\
\hline Estado Civil & $\begin{array}{l}\text { Soltero: } 90,84 \% \text { Casado: } 2,05 \% \text { Separado: 0,32\% } \\
\text { Madre Soltera: 0,16\% Unión Libre: } 6,64 \%\end{array}$ \\
\hline Ciudad de Residencia & Bogotá: 89,10\% Otra Ciudad: 10,90\% \\
\hline Estrato & $\begin{array}{l}\text { Estrato 1: 10,90\% Estrato 2: } 38,86 \% \text { Estrato 3: } 44,23 \% \\
\text { Estrato 4: 5,90\% Estrato 5: 0,63\% Estrato 6: 0,16\% }\end{array}$ \\
\hline Resultados Saber 11 & $\begin{array}{l}\text { Media: } 467 \text { Mínimo: } 100 \text { Máximo: } 992 \\
\text { Estudiantes ubicados en el cuartil 1: 25,7\% }\end{array}$ \\
\hline Tipo de Colegio & Público: 49,13\% Privado: 50,87\% \\
\hline
\end{tabular}

Fuente: Elaboración propia.

\section{Análisis de Supervivencia-Kaplan Meier}

Como primera parte del análisis de la permanencia de los estudiantes se estima el riesgo de deserción presentado en cada uno de los momentos del tiempo para lo cual se recurre al análisis de supervivencia de Kaplan Meier. El objetivo de la metodología de KaplanMeier (KM) es estimar la probabilidad de supervivencia (para este caso de permanencia) de un grupo de personas en un intervalo de tiempo definido (probabilidad condicionada). KM utiliza una función de supervivencia no-paramétrica para un grupo de personas (en otras palabras, su probabilidad de supervivencia después del momento t) y por tanto no hace ninguna sunción sobre la distribución de supervivencia. La característica distintiva es que la proporción acumulada que sobrevive se calcula para el tiempo de supervivencia individual de cada persona y no se agrupan los tiempos de supervivencia en intervalos (Arribalzaga, 2007).

Para este estudio se utiliza el paquete estadístico de Stata 11.0 para calcular la función de supervivencia con los estimadores de KM.

\section{Modelo de duración para el análisis de la deserción estudiantil}

Los modelos de duración describen el comportamiento de datos que corresponden al tiempo o duración que tarda en ocurrir un evento, que para estecaso es la deserción estudiantil a partir de un inicio definido (Díaz, 2009) (Giovagnoli, 2002) (Herrero, Merlino, Ayllon \& Escanes, 2013) (Ministerio de Educación Nacional, 2009). Un modelo de duración se enfoca en determinar la probabilidad condicional de que un evento ocurra, y 
se condiciona al tiempo que ha durado el evento. Aplicado al análisis de la permanencia estudiantil la supervivencia o retención del estudiante corresponderá a la probabilidad de que el estudiante permanezca dado que no ha desertado hasta el último periodo observado. Adicionalmente, estos modelos permiten incluir factores explicativos en el tiempo por lo que se convierte en una posibilidad de analizar variables que pueden determinar la decisión de abandono en un momento dado (Giovagnoli, 2002).

El modelo de duración fue escogido para el análisis de los datos utilizados en este estudio debido a la validación y uso que ha tenido esta herramienta estadística en temas relacionados con la permanencia estudiantil. De acuerdo al Ministerio de Educación Nacional de Colombia (2009), los modelos de duración son los más adecuados para analizar la permanencia estudiantil ya que éstos permiten estimar el riesgo que un estudiante deserte teniendo en cuenta tanto las características asociadas al estudiante como el tiempo de permanencia en la institución. De este modo, la conveniencia de los modelos de duración se da por la relación entre temporalidad y la variación de las condiciones, la estimación de la probabilidad de que el abandono ocurra en cierto periodo, el análisis del periodo de riesgo y de las tasas de ocurrencia del evento, y la admisión de observaciones con censura (Escanes, Herrero y Merlino, 2013).

Específicamente, para este estudio se utilizó el modelo de Cox de riesgos proporcionales con el fin de evaluar las variables escogidas para ver su relación con el riesgo de deserción estudiantil. Esta regresión tiene en cuenta el orden en el que los eventos se producen en el tiempo, realizando una estimación de riesgo de que suceda un evento en un momento temporal aleatorio $t+1$ (que el estudiante deserte), condicionado a que el estudiante haya permanecido hasta el momento t (Cox, 1972). Un modelo de Cox estima los cocientes de riesgos, que miden qué tanto una variable afecta la función de riesgo para el evento de interés (deserción). De acuerdo con la suposición de riesgos proporcionales, las variables actúan multiplicativamente sobre una función de riesgo de referencia (Royston y Altman, 2013).

Para el tratamiento de los datos se ha recurrido al paquete estadístico Stata (versión 11.0).

\section{REsultados}

Como se mencionó en la metodología propuesta para el estudio, primero se calcula la función de supervivencia (permanencia) utilizando la metodología de Kaplan Meier. Para tal fin se utiliza el comando de Stata correspondiente y que permite ver la curva de probabilidades de que un estudiante permanezca en su carrera. Según los resultados de supervivencia, los estudiantes tienen una mayor probabilidad de deserción en los primeros periodos académicos con un riesgo de 30,5\% para el primer periodo y para el primer año el riesgo alcanza un valor de 61,3\%. Este primer ejercicio arroja que el $30,6 \%$ de los estudiantes son los que logran culminar sus estudios técnicos profesionales (Figura 1). 


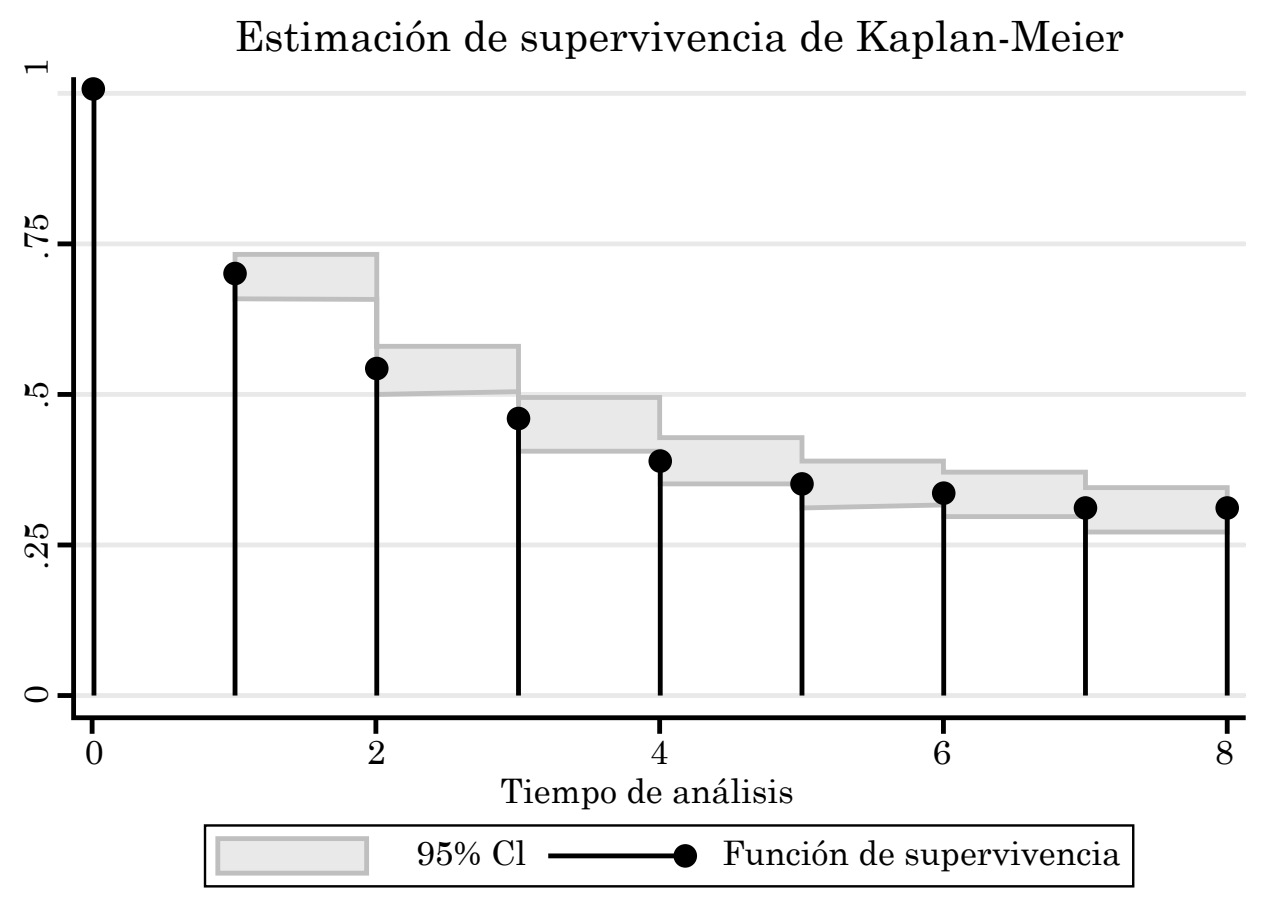

Figura 1. Función de supervivencia Kaplan Meier. Fuente: Elaboración propia.

Con el panorama anterior, lo que sigue es entender la correlación de las variables interés y su efecto sobre la permanencia estudiantil (variable independiente). Como se menciona en las consideraciones metodológicas, se realiza un análisis bajo el modelo de riesgo propuesto por Cox (Diaz, 2009) (Giovagnoli, 2002) (Herrero, Merlino, Ayllon, \& Escanes, 2013) (Ministerio de Educación Nacional, 2009). Para correr el modelo se utiliza Stata 11.0 donde se incluyen los parámetros indicados por el programa para el análisis de Cox. El análisis arroja una comprobación acerca de que los datos están correctamente planteados en términos de un modelo de duración y asimismo como resultado se generan 4 variables adicionales que se utilizan para la estimación del riesgo:_st que indica si la observación se incluirá o no en el análisis (1 se incluye, 0 se excluye), _d que indica el balance entre observaciones censuradas y no censuradas (1 no censura, 0 censura), _t registra el momento en que acaba el estado y t0 registra el momento en que se inicia el estado.

Tras correr el modelo se obtienen los resultados que se observan en la tabla 4, donde se incluyen los ratios de hazard, la significancia obtenida y el intervalo de confianza. En primer lugar, se observa que la edad tiene una influencia significativa sobre el modelo $(p=0,000)$ y por lo tanto se convierte en un primer predictor de la permanencia estudiantil. Así es como, el riesgo de desertar de quienes se encuentran en el primer cuartil de edad es 50\% más de los que tienen otra edad. Respecto a las variables de género, estado civil y ciudad de residencia se encuentra que no existen diferencias significativas entre los puntos de comparación propuestos. Sin embargo, si hay una diferencia encontrada entre los estudiantes pertenecientes a los estratos 1 y 2 en comparación con los demás estratos $(\mathrm{p}=0,002)$. De esta forma se encuentra que el riesgo de desertar de quienes pertenecen a los estratos 1 y 2 es $25 \%$ más de los que se encuentran en estratos superiores. Finalmente, no se puede comprobar ningún efecto sobre la permanencia estudiantil en cuanto al puntaje obtenido de las pruebas de estado Saber 11 y el tipo de colegio del que proviene el estudiante (Tabla 5). 
TABLA 5.

Resultados de estimación del riesgo con el modelo de Cox.

\begin{tabular}{lcccccc}
\hline \multicolumn{1}{c}{ Variable } & Haz. Ratio & Std. Err. & $\mathrm{z}$ & \multicolumn{2}{c}{ P > I z I } & \multicolumn{2}{c}{ [95\% Conf. Interval] } \\
Edad & 1.501948 & .1501795 & 4.07 & 0.000 & 1.234648 & 1.827117 \\
Femenino & .8986533 & .0880269 & -1.09 & 0.275 & .7416744 & 1.088857 \\
Soltero & .7462762 & .1212364 & -1.80 & 0.072 & .5427722 & 1.026081 \\
ResBogotá & 1.257239 & .2059901 & 1.40 & 0.162 & .9119147 & 1.733331 \\
Estrato12 & 1.246163 & .1233063 & 2.22 & 0.002 & 1.026477 & 1.512865 \\
Saber11 & 1.077899 & .1183816 & 0.68 & 0.495 & .8691477 & 1.336787 \\
Cole Público & 1.07198 & .1053608 & 0.71 & 0.479 & .8841492 & 1.299715 \\
\hline
\end{tabular}

Fuente: Elaboración propia.

\section{DisCUSIÓN Y CONSIDERACIONES A PARTIR DE LOS RESULTADOS}

De la función de supervivencia se puede ver que el riesgo de deserción es alarmante en cuanto a su cantidad absoluta y solo el $30,6 \%$ de los estudiantes alcanzan su grado. No obstante, este dato no está por fuera de la realidad nacional donde para las carreras técnicas profesionales el índice de graduación oscila en un 39\% sobre la base de los estudiantes de primer ingreso. La función también permite observar que el riesgo de deserción es menor a medida que el tiempo avanza y podría inferirse que las estrategias deberían concentrarse en los primeros periodos donde se concentra el mayor riesgo (61,3\% para el primer año). Esta realidad confirma el reto que tienen las instituciones de educación superior en el nivel de formación técnica para brindar condiciones que favorezcan la permanencia estudiantil y mejoren la tasa de graduación en los indicadores de educación superior.

En cuanto a las variables de ingreso que pueden relacionarse con la permanencia estudiantil se pueden tener varias conclusiones que apoyan y otras difieren con lo encontrado en investigaciones previas. Por su parte, la primera variable estadísticamente significativa sobre la permanencia de los estudiantes es la edad, para lo cual se obtiene que los estudiantes más jóvenes (15 - 18 años) tienen una mayor probabilidad de abandonar sus estudios que las demás personas con mayor edad. Esta información no es compatible con lo expuesto por Giovagnoli (2002) que ofrece un resultado parcialmente opuesto, donde se encuentra que los estudiantes con mayor edad tienen mayor propensión a abandonar sus estudios. Sin embargo, los resultados arrojados en esta investigación presentan otra perspectiva para el contexto específico analizado.

Algunas hipótesis que resultan acerca de las correlaciones analizadas reúne explicaciones como: las personas más jóvenes no tienen claridad sobre sus decisiones al salir del colegio y puede provocar cambios en las primeras etapas postsecundaria; las personas más jóvenes pueden ser influenciadas en sus decisiones por las personas más cercanas y que pueden no corresponder a los intereses reales del estudiante; os estudiantes recién egresados de la educación secundaria no han desarrollado plenamente la competencia de resiliencia por lo 
que ante situaciones problemáticas pueden fácilmente frustrarse y abandonar un proyecto. Asociado a lo anterior, las bases de la educación media vienen muy deficientes sin oportunidad de haberlas superado lo que puede causar efectos negativos sobre los resultados académicos. También, la dependencia económica de los padres puede ser otro factor que influya en el abandono si la situación familiar cambia (lo cual es muy común en estratos bajos).

Por otro lado, en correspondencia al estudio de Herrero (Herrero, Merlino, Ayllon, \& Escanes, 2013) se encuentra una relación estadísticamente significativa entre el estrato social y la permanencia de los estudiantes. Los estudiantes que pertenecen a los estratos más bajos ( 1 y 2 ) tienen una mayor probabilidad de abandonar sus estudios. Esta situación se puede explicar ya que los estudiantes de estratos más altos vienen de hogares con padres más escolarizados y/o con mejor inserción laboral (Ishitani \& DesJardins, 2002). De acuerdo a los antecedentes de la institución objeto del estudio, las dificultades económicas son la causa con mayor frecuencia que reportan los estudiantes, lo cual podría estar relacionado con el estrato social.

Finalmente, se espera que estos hallazgos puedan brindar a la Institución donde se realiza el estudio y a otras instituciones, un aporte para derivar acciones enfocadas a prevenir la deserción estudiantil. Algunas ideas que surgen de la socialización de los resultados al interior de la Institución incluyen las siguientes: - Fortalecer el plan de orientación profesional cuando el estudiante está en proceso de selección de su carrera para promover una decisión más consciente y acertada. -Implementar estrategias de acompañamiento profesional para atender la necesidad de fortalecer la capacidad de afrontamiento a las dificultades que se puedan presentar. -Consolidar estrategias de nivelación para las competencias básicas (matemáticas, lectura, escritura, informática). - Proveer a los estudiantes posibilidades de ingresos económicos para solventar sus gastos y el pago de las matrículas (bolsa de empleo, becas, eventos para recolectar fondos).

\section{REFERENCIAS}

Abarca, A. \& Sanchez, M. (2005). La Deserción Estudiantil en la Educación Superior: El Caso de la Universidad de Costa Rica. Actualidades Investigativas en Educación, 5, 1-22. Disponible en https://www.redalyc.org/pdf/447/44759911.pdf

Arribalzaga, E. (2007). Interpretación de las Curvas de Supervivencia. Revista Chilena de Cirugía, 59(1), 75-83. Disponible en https://www.redalyc.org/pdf/3455/345531922013. pdf

Cox, D. (1972). Models and Life-Tables. Journal of the Royal Statistical Society, 34(2), 187 220. Disponible en http://www.biecek.pl/statystykaMedyczna/cox.pdf

Díaz, C. (2009). Factores de Deserción Estudiantil en Ingeniería: Una Aplicación de Modelos de Duración. Información Tecnológica, 20(5), 129-145. Disponible en http://www. biecek.pl/statystykaMedyczna/cox.pdf

Enciso, J., Córdoba, L. y Romero, L. (2016). Adaptaciones curriculares para el ingreso, permanencia y graduación de estudiantes con discapacidad: una experiencia desde la Educación Superior. Cultura Educación y Sociedad, 7(2), 72-93 Disponible en https:// revistascientificas.cuc.edu.co/culturaeducacionysociedad/article/view/1103 
Escanes, G., Herrero, V. \& Merlino, A. (2013). El Estudio de la Permanencia de Estudiantes en la Universidad: Elementos para la Aplicación de un Modelo de Duración. Cuadernos de Educación, (11), 1-14.Disponible en https://ri.conicet.gov.ar/handle/11336/8475

Giovagnoli, P. (2002). SEDICI. Repositorio Institucional de la UNLP. Recuperado de http:// sedici.unlp.edu.ar/handle/10915/37129

Gold, J. (1995). An Intergenerational Approach to Student Retention. Journal of College Student Development, 36(2), 182-187. Diponible en https://psycnet.apa.org/record/1995-35006-001

González, M., Álvarez, P., Cabrera, L. \& Bethencourt, J. (2007). El Abandono de los Estduis Universitario: Factores Determinantes y Medidas Preventivas. Revista Española de Pedagogía, (236), 71-86. Disponible en https://revistadepedagogia.org/wp-content/uploads/2007/06/236-07.pdf

Herrero, V., Merlino, A., Ayllon, S. \& Escanes, G. (2013). Aplicación de un Modelo de Duración en Programas de Prevención de Deserción Universitaria. Revista Electrónica de Investigación Educativa, 15(3), 38-52. Disponible en http://www.scielo.org.mx/pdf/ redie/v15n3/v15n3a3.pdf

Huesca, G. \& Castaño, M. (2007). Causas de Deserción de Alumnos de Primeros Semestres de una Universidad Privada. REMO, 5(12), 34-39. Disponible en https://ridda2. utp.ac.pa/bitstream/handle/123456789/9377/1319582164causas-de-desercion-en-unauniversidad-privada35.pdf?sequence $=1 \&$ isAllowed $=y$

Ishitani, T. \& DesJardins, S. (2002). A longitudinal investigation of dropout from college in the United States. Journal of College Student Retention, 4(2), 173-201. Disponible en https://journals.sagepub.com/doi/10.2190/V4EN-NW42-742Q-2NTL

Luque, M. y Marín, F. (2001). Inserción de los proyectos pedagógicos de aula en las prácticas profesionales de la docencia. Multiciencias, 1 (2), 117-128. Disponible en https:// www.redalyc.org/pdf/904/90412006.pdf

Pascarella, E. \& Terenzini, P. (1983). Predicting voluntary freshman year persistence / withdrawal behavior in a residential university: A path analytic validation of Tinto's model. Journal of Educational Psychology, 75(2), 215-266. Disponible en https://scielo.conicyt.cl/scielo.php?script=sci_nlinks\&ref=3426723\&pid=S07180764201600060000200009\&lng=es

Patiño, L. \& Cardona, A. (2012). Revisión de Algunos Estudios sobre la Deserción Estudiantil Universitaria en Colombia y Latinoamerica. Theoria, 21(1), 9-20. Disponible en http://www.ubiobio.cl/miweb/webfile/media/194/v/v21-1/1.pdf

República de Colombia. Ministerio de Educación Nacional. (2015). Sistema Nacional de Información de la Educación Superior. Recuperado de http://www.mineducacion.gov. co/sistemasdeinformacion/1735/articles-358471_recurso_4.pdf

República de Colombia. Ministerio de Educación Nacional. (2009). Sistema Nacional de Información de la Educación Superior. Recuperado de http://www.mineducacion.gov. co/sistemasdeinformacion/1735/articles-358471_recurso_9.pdf

Royston, P. \& Altman, D. (2013). External validation of a Cox prognostic model: principles and methods. BMC Medical Research Methodology, 13-33. Disponible en https://bmcmedresmethodol.biomedcentral.com/articles/10.1186/1471-2288-13-33 
Rue, J. (2014). El Abandono Universitario: Variables, Marcos de Referencia y Políticas de Calidad. Revista de Docencia Universitaria, 12(2), 281-306. Disponible en https:// polipapers.upv.es/index.php/REDU/article/view/5649

Tinto, V. (1993). Reflexiones sobre el Abandono de los Estudios Superiores. Perfiles Educativos, (62), 56-63. Disponible en http://www.iisue.unam.mx/perfiles/busqueda. php?indice=autor\&busqueda=TINTO,\%20VINCENT

\footnotetext{
Notas al final

1 El Politécnico Internacional ofrece carreras de nivel técnico profesional con una duración de 2 años en periodos académicos trimestrales.

2 Examen de Estado Colombiano de la educación media que nace en 1968 con el propósito de apoyar a las instituciones de educación superior en sus procesos de selección y admisión de estudiantes.

3 El Politécnico Internacional ofrece carreras de nivel técnico profesional con una duración de 2 años en periodos académicos trimestrales.

4 Examen de Estado Colombiano de la educación media que nace en 1968 con el propósito de apoyar a las instituciones de educación superior en sus procesos de selección y admisión de estudiantes.
} 